\title{
Mortality by Relative Weight and Exercise
}

\author{
LAWRENCE GARFINKEL, MA, AND STEVEN D. STELLMAN, PHD
}

\begin{abstract}
Mortality in relation to relative weight and exercise was analyzed from data collected in the first 2 years of the American Cancer Society's Cancer Prevention Study II. It included data on 868,620 persons who had no history of cancer, heart disease, or stroke and who were not sick at the start of the study. Standardized mortality ratios (SMR) were 187 for men who were $20 \%$ or more underweight and 187 for men who were $40 \%$ or more overweight. Comparable SMR in women were 186 and 178 . Current smokers had considerably higher SMR than nonsmokers for all causes of death and cancer deaths at each relative weight category. Degree of exercise reported by subjects was inversely related to mortality, independent of smoking habits. Subjects who exercised heavily and died of cancer of the lung, colonrectum, and pancreas had $20 \%$ to $37 \%$ higher SMR than men and women who reported moderate exercise.
\end{abstract}

Cancer 62:1844-1850, 1988.

A NUMBER OF STUDIES have looked at body weight in relation to mortality. The results of 25 studies reviewed by Manson et al. ${ }^{1}$ were discrepant. Ten showed no association; in those that did show an association with body weight, the shape of the curves of relative risk varied. But two of the largest studies, including one by the American Cancer Society, ${ }^{2,3}$ did show similar weight-mortality curves that had a $\mathrm{J}$ shape; that is, slightly higher relative risks were observed for underweight subjects compared to subjects of average weight and high relative risks were observed for overweight subjects. The studies showed that the lowest mortality rates were at relative weights $5 \%$ to $10 \%$ below average weight. Manson et al. ${ }^{1}$ emphasized that three factors were important in evaluating body weight in relation to mortality: cigarette smoking habits, hypertension and hyperglycemia, and subclinical disease.

In the American Cancer Society study relative risks for total mortality in men ranged from 1.25 in men $20 \%$ or more under weight to 1.87 in men $40 \%$ or more overweight compared to a relative risk ratio of 1.00 for average weight men. In women the comparable mortality ratios were 1.19 in underweight women and 1.89 for overweight women. Cancer risk ratios ranged from 1.33 in underweight men to 1.55 for overweights. In women the ratios for cancer were 0.96 to 1.55 , respectively.

Presented at the American Cancer Society Second National Conference on Cancer Prevention and Detection, Seattle, Washington, June 25-27, 1987.

From the Department of Epidemiology and Statistics, American Cancer Society, New York.

Address for reprints: Lawrence Garfinkel, MA, American Cancer Society, 1180 Avenue of the Americas, New York, NY 10001

Accepted for publication September 24, 1987.
Colon-rectum cancer and prostate cancer death rates were elevated in overweight men and rates for cancer of the endometrium, cervix, gall bladder, ovary, and breast were elevated in obese women.

\section{Materials and Methods}

Cancer Prevention Study II (CPS II) is a prospective study of death rates from cancer and other causes of death. In the fall of 1982 more than 77,000 American Cancer Society volunteer researchers enrolled over 1.2 million men and women in a long-term prospective study. Those enrolled, who usually were friends, neighbors, or relatives of the volunteers, completed a fourpage confidential questionnaire on current illness, history of cancer and other diseases, occupational exposures, use of medicines and vitamins, menstrual and reproductive history, and diet and drinking habits including smoking. Enrollment was by family groups with at least one person in the household 45 years of age or older. All members of the household older than 30 years were asked to complete a questionnaire.

Enrollment of subjects was carried out in all 50 states, the District of Columbia, and Puerto Rico. It began in September 1982 and was essentially completed by the end of November 1982. The average age of the subjects was 58 and about $94 \%$ were white. Thirty-six percent of the men and $25 \%$ of the women had a college education or graduate school education.

Every second year, researchers are sent a list of the persons enrolled and asked to check whether those enrolled are alive or dead. For deaths, the researchers record the date and place of death. Death certificates 
TABLE 1. Percentage Distribution of Subjects and Deaths by Health Status at Start of Study

\begin{tabular}{|c|c|c|c|c|}
\hline \multirow[b]{2}{*}{ Health status at baseline } & \multicolumn{2}{|c|}{ Men } & \multicolumn{2}{|c|}{ Women } \\
\hline & $\begin{array}{c}\text { All subjects } \\
(\%)\end{array}$ & $\underset{(\%)}{\text { Dead in } 2 \mathrm{yr}}$ & $\begin{array}{c}\text { All subjects } \\
(\%)\end{array}$ & $\begin{array}{c}\text { Dead in } 2 \mathrm{yr} \\
(\%)\end{array}$ \\
\hline Sick,*history† & 4.0 & 24.5 & 3.2 & 26.5 \\
\hline Well, history & 12.8 & 27.6 & 11.6 & 26.9 \\
\hline Sick, no history & 5.5 & 9.1 & 7.0 & 8.9 \\
\hline Well, no history & 77.7 & 38.8 & 78.2 & 37.8 \\
\hline Total & 100.0 & 100.0 & 100.0 & 100.0 \\
\hline
\end{tabular}

* Answered yes to the question, Are you sick at the present time?

$†$ Reported history of cancer, heart disease, or stroke.

subsequently are obtained through state health departments.

The first follow-up was conducted in 1984 and we report here a preliminary analysis based on death rates in the first 2 years of the study for selected causes of death in relation to relative weight at enrollment and in relation to self-reported exercise habits.

Relative weight for each subject is defined as that person's weight divided by the average weight of persons of the same sex, age and height. Age was taken in 5-year age groups. Height categories included single inches of height in men from 60 inches to 79 inches. Height categories in women were 54 to 73 inches. Subjects were placed into seven relative weight classes: $<80,80$ to 89 , 90 to 104,105 to 119,120 to 129,130 to 139 , and $140+$.

The relation between relative weight and mortality was evaluated using standardized mortality ratios (SMR). Person-years of exposure (PYE) to risk of dying were accumulated for each individual, beginning on the date of enrollment and continuing until the 2-year cutoff if alive, or to date of death. The PYE were stratified by 5 -year age groups and by relative weight category. Age-specific rates were calculated by dividing the observed deaths in the referent category $(90-104)$ by PYE and multiplying by the age-specific PYE of each relative weight category. The results were summed over age to provide the number of estimated deaths (E) in each relative weight category. The observed number of deaths $(\mathrm{O})$ is divided by $\mathrm{E}$ and multiplied by 100 to obtain the SMR. Since weight often is related to serious illness, it was necessary in this analysis to exclude persons who had a history of cancer, heart disease, or stroke which could cause weight loss, since inclusion of such persons could artificially increase the SMR for underweight persons. In addition we excluded persons who answered "yes" to the question, "Are you sick at the present time?." We are not certain if people with a history of other diseases also had a loss of weight because of their condition; but in this study, subjects classified as having a history of cancer, heart disease, or stroke or "sick at present" had death rates three times as high as those who did not report any of these conditions.

Subjects in our study were asked the question, "How much exercise do you get (work or play)?" The choices were none, slight, moderate, or heavy. An analysis also was made of mortality in relation to exercise patterns.

\section{Results}

In the first 2 years of this study 11,489 men and 7688 women were reported dead. Death certificates have been received thus far for $96.3 \%$ of the deaths. Thirty-three percent of the male deaths and $41.3 \%$ of the female deaths were of cancer. Lung cancer was the most common site in men $(33 \%)$ followed by colon-rectum cancer $(13.1 \%)$ and pancreatic cancer $(7.0 \%)$. Breast cancer was the leading cause in women $(25.3 \%)$ followed by lung cancer $(15.0 \%)$ and colon-rectum cancer (12.7\%).

Table 1 shows the percentage distribution of CPS II subjects and deaths classified by whether or not they said they were "sick" and whether or not they had a history of heart disease, stroke or cancer. Whereas only $4.0 \%$ of all male subjects had such a history and also said they were sick at the start of the study, $24.5 \%$ of all men who died within the next 2 years were in that category. Similar results held for women. On the other hand about $78 \%$ of the subjects had no history and were not sick, and only $38 \%$ of the deaths were in this category.

For this study, 868,620 persons $(372,336$ men and 496,284 women) met the criteria that height and weight were recorded within the parameters defined above and had no history of heart disease, stroke or cancer, and did not report "sick at present." In this group 7305 deaths were reported in the first 2 years of the study ( 4440 men and 2865 women) and are the subjects for this study.

Figure 1 shows the SMR by relative weight and state of health at enrollment. The SMR for men and women with relative weight of $<80$ who were sick or had serious diseases were nearly three times as high as for average weight subjects, and eight or nine times as high as per- 


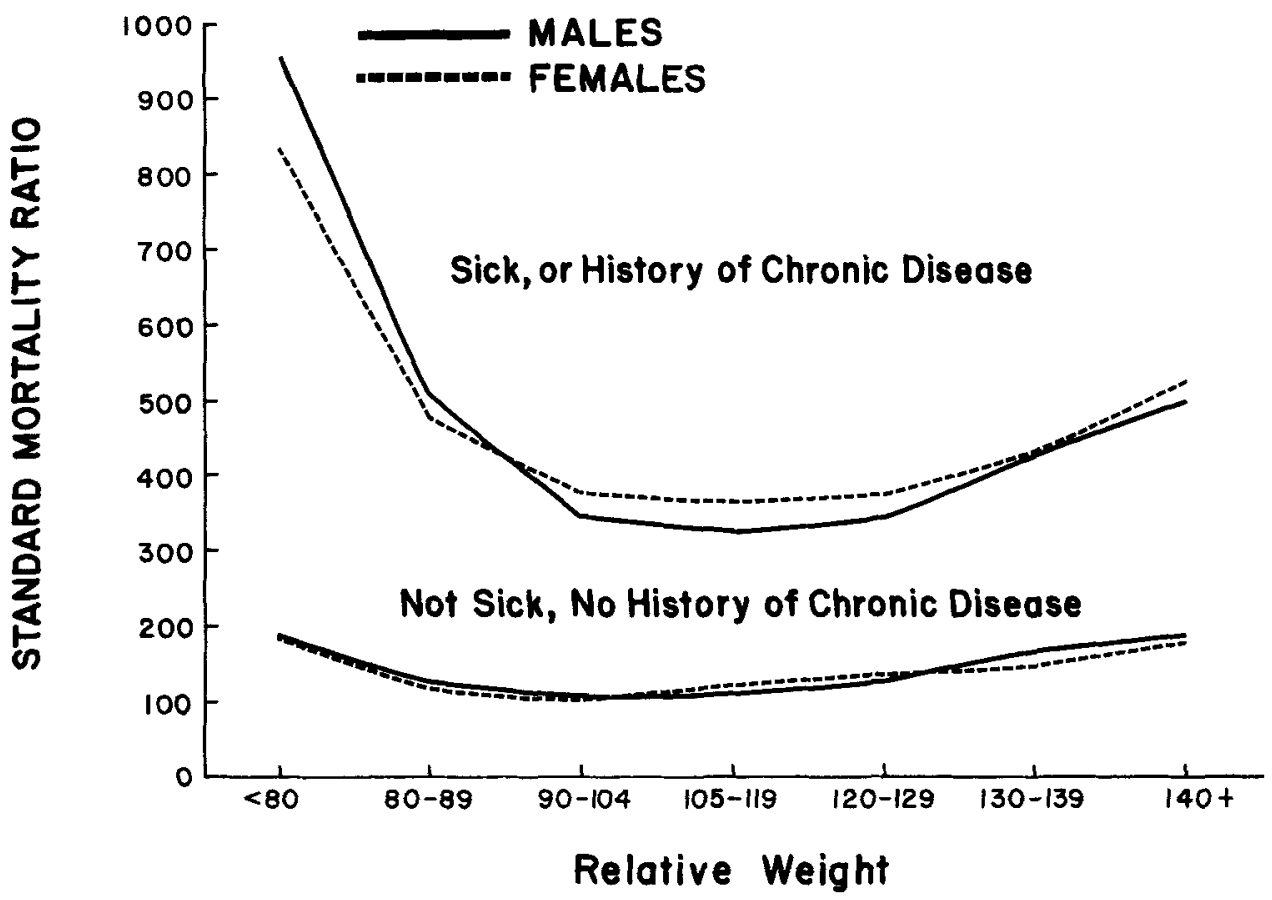

FIG, 1. Standard mortality ratios by relative weight for all causes of death by disease history.

sons of average weight who were not sick and had no history of serious disease. This graphically justifies exclusion of such people from further analysis.

In those who were neither sick nor had a history of serious disease, the curve was $U$ shaped, not $J$ shaped as in the first American Cancer Society Study (Fig. 1). The SMR were 187 in men $20 \%$ or more underweight and 171 for those $40 \%$ or more overweight; in women the comparable SMR were 186 and 178. The lowest mortality rate was in the referent category (relative weight, 90-104). Table 2 also shows the SMR for relative weight categories by age group. In both men and women the relative risks were mostly lower in relative weight cate-

TABle 2. Standardized Mortality Ratios by Relative Weight by Age and Sex

\begin{tabular}{ccccccc}
\hline & \multicolumn{5}{c}{ Relative weight } \\
\cline { 2 - 7 } Sex/age & $<80$ & $80-90$ & $90-104$ & $105-119$ & $120-129$ & $130+$ \\
\hline Men & & & & & & \\
$40-49$ & $197^{*}$ & 115 & 100 & 121 & 178 & 193 \\
$50-59$ & 143 & 115 & 100 & 112 & 126 & 138 \\
$60-69$ & 221 & 134 & 100 & 116 & 110 & 203 \\
$70-79$ & 197 & 121 & 100 & 102 & 137 & 182 \\
$80+$ & 189 & 147 & 100 & 97 & 98 & 120 \\
Women & & & & & & \\
$40-49$ & 137 & 81 & 100 & 137 & 156 & 150 \\
$50-59$ & 142 & 100 & 100 & 116 & 154 & 135 \\
$60-69$ & 198 & 116 & 100 & 119 & 147 & 140 \\
$70-79$ & 193 & 131 & 100 & 137 & 122 & 186 \\
$80+$ & 211 & 141 & 100 & 96 & 110 & 188 \\
\hline
\end{tabular}

* Based on less than ten observed deaths. gories $<80$ and 80 to 89 in those younger than 60 years than in those 60 years and older. In other words, extreme underweight was a serious factor mainly in subjects older than 60 years. Mortality rates were relatively low in men older than age 80 who were above average weight.

Table 3 shows SMR by relative weight for other selected causes. Ischemic heart disease is low in underweight men and rises to 222 for men $40 \%$ or more overweight. Women show a similar pattern, although SMR are higher for underweights. Cerebrovascular disease is high for underweights in both sexes, is lowest for average weight persons, and increases in overweights. Cancer of all sites has SMR of more than 200 in men for extreme underweights $(<80)$ and overweights $(140+$ pounds). Women have the same pattern but with an SMR of 163 in relative weight category $<80$, and 148 in obese women. The SMR for lung cancer in underweights $(<80)$ is more than 250 for both men and women and less than 100 for persons with relative weight of 105 or greater. The SMR for colon-rectum cancer show no definable pattern in women. In men the SMR are higher in underweight and overweight men, but they are based on small number of deaths.

Figure 2 shows SMR by relative weight by smoking habits for men and women. Because of relatively low death rates in the first 2 years of the study, the category "nonsmokers" is defined as lifelong nonsmokers plus exsmokers who quit 10 or more years before the study began. Death rates for these two groups were very simi- 
TABLE 3. Standardized Mortality Ratios by Relative Weight for Selected Causes of Death, by Sex

\begin{tabular}{|c|c|c|c|c|c|c|c|c|c|}
\hline \multirow[b]{2}{*}{ Sex/cause of death } & \multirow[b]{2}{*}{ ICD-9 codes } & \multirow[b]{2}{*}{$\mathbf{N}$} & \multicolumn{7}{|c|}{ Relative weight } \\
\hline & & & $<80$ & $80-89$ & $90-104$ & $105-119$ & $120-129$ & $130-139$ & $140+$ \\
\hline \multicolumn{10}{|l|}{ Men } \\
\hline Ischemic heart disease & $410-414$ & 1502 & 97 & 97 & 100 & 111 & 159 & 199* & 222 \\
\hline Cerebrovascular disease & $430-438$ & 232 & 282 & 132 & 100 & 133 & 165 & 235 & 157 \\
\hline All cancer & $140-208$ & 1355 & 219 & 131 & 100 & 98 & 84 & 140 & 225 \\
\hline Lung cancer & 162 & 507 & 266 & 161 & 100 & 83 & 76 & $63^{*}$ & $91^{*}$ \\
\hline Colorectal cancer & 153,154 & 150 & $178^{*}$ & 110 & 100 & 100 & $62^{*}$ & $205^{*}$ & $205^{*}$ \\
\hline \multicolumn{10}{|l|}{ Women } \\
\hline Ischemic heart disease & $410-414$ & 702 & 131 & 105 & 100 & 111 & 134 & 199 & 212 \\
\hline Cerebrovascular disease & $430-438$ & 290 & 227 & 104 & 100 & 107 & 130 & 131 & 142 \\
\hline All cancer & $140-208$ & 992 & 163 & 123 & 100 & 120 & 113 & 140 & 148 \\
\hline Lung cancer & 162 & 220 & 253 & 153 & 100 & 87 & $62^{*}$ & $54^{*}$ & $31^{*}$ \\
\hline Colorectal cancer & 153,154 & 133 & $98^{*}$ & 91 & 100 & 103 & 121 & $76^{*}$ & $65^{*}$ \\
\hline
\end{tabular}

* Less than five observed deaths.

lar. The referent category for each sex was the nonsmokers with relative weight 90 to 104 . Current smokers had rates considerably higher than nonsmokers irrespective of relative weight. In women the SMR in the relative weight category $130+$ was unexpectedly low due probably to the small number of observed deaths and resulting instability of death rates.

Weight and exercise are interrelated to some degree, and it is possible that exercise does affect SMR for relative weight categories. A separate analysis of relative weight therefore was made for current smokers and nonsmokers restricted to subjects who reported they exercised moderately or heavily. The same pattern of high relative mortality ratios for underweights and overweights was seen as shown in Figure 2, except that they were at a lower absolute level. In male smokers, for example, the SMR were 336 and 314 for relative weight categories $<80$ and $130+$, respectively; compared to 377 and 380 , respectively, for all subjects. In male nonsmokers the SMR were 145 and 172 for moderate or heavy exercisers, respectively, compared to 158 and 190 . Females showed the same relative patterns of mortality.

The influence of weight on cancer death rates is similar to that for all causes, as shown in Figure 3. The curves are more broken due to smaller numbers of observations. Higher mortality ratios are observed for extreme underweights (relative weight $<80$ ) and overweights (130+) among male smokers, but in general, the same mortality curves are present as for all causes of deaths.

One of the questions in the questionnaire was, "How much exercise do you get (work or play)?" The choices were none, slight, moderate, or heavy. Figures 4 and 5 are concerned with the relation between exercise and
FIG. 2. Standardized mortality ratios by relative weight for all causes of death, for current smokers and nonsmokers, by sex. *SMR based on only 12 deaths.

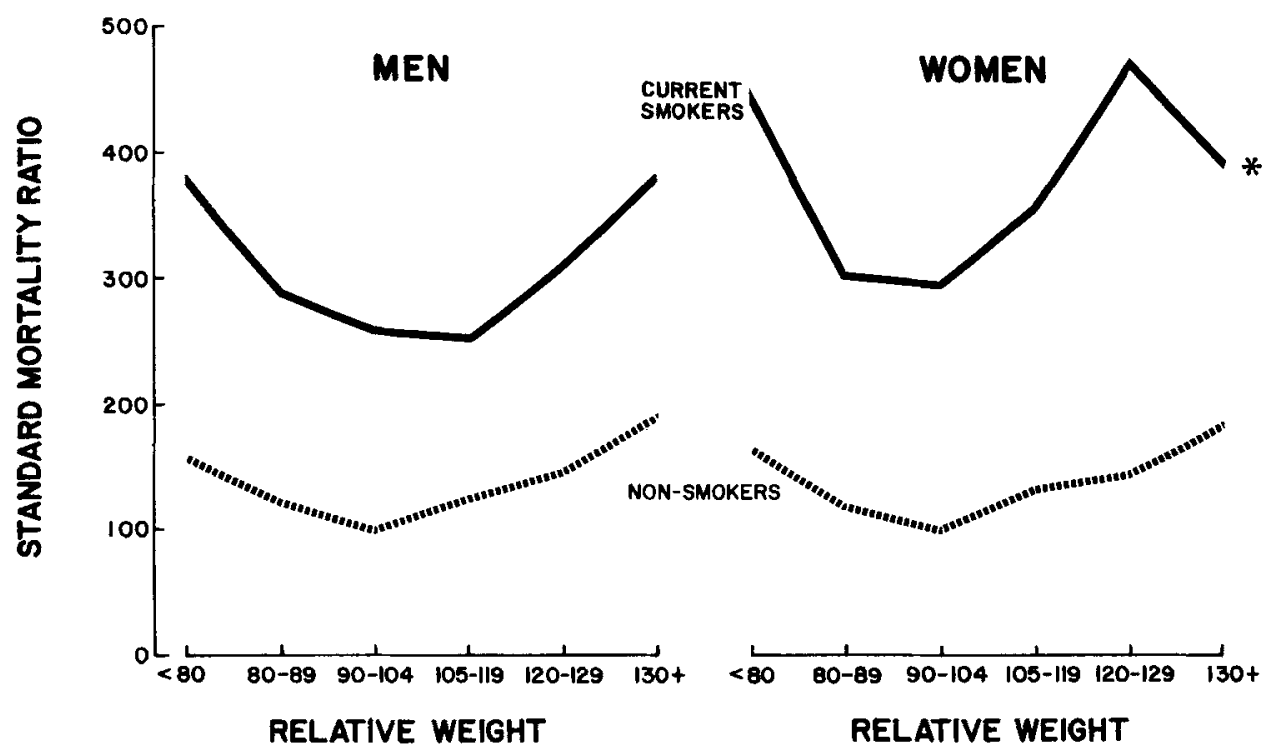




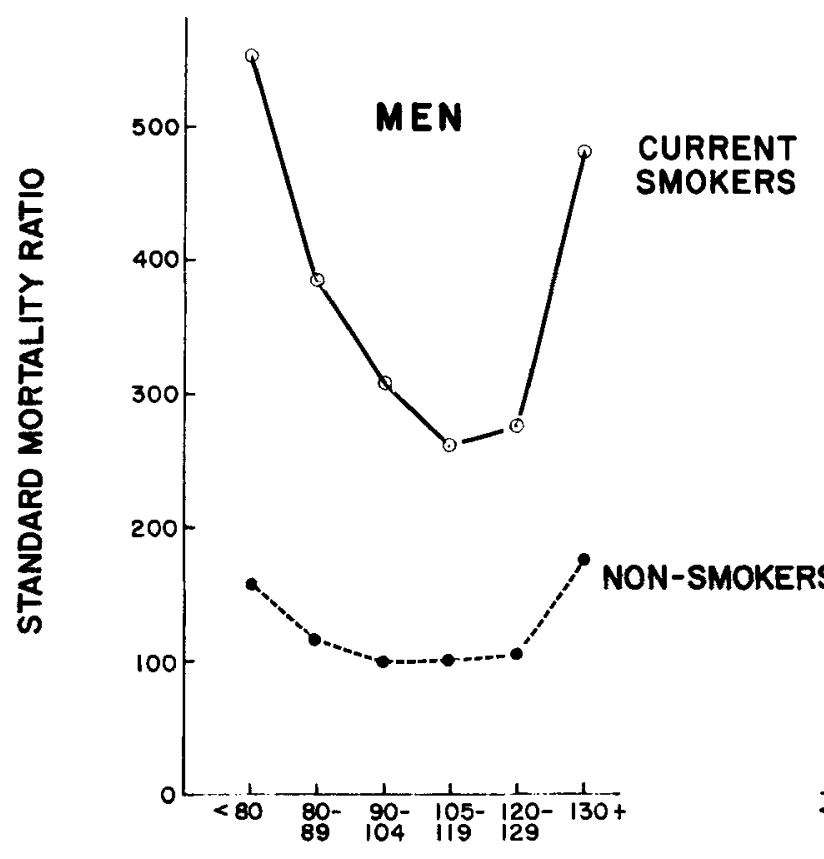

RELATIVE WEIGHT

\section{WOMEN}
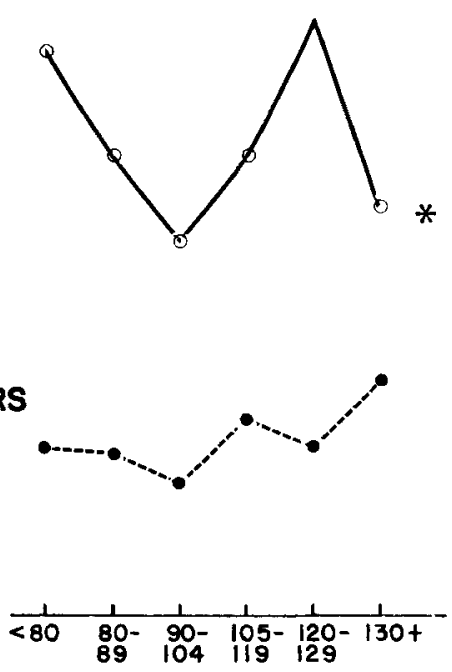

RELATIVE WEIGHT
FIG. 3. Standardized mortality ratios by relative weight for cancer (all sites), for current smokers and nonsmokers, by sex. "SMR based on only three deaths. death rates in those who had no history of heart disease, stroke, or cancer and were not sick at the start of the study. Data are shown for current smokers and nonsmokers (as previously defined). Figure 4 shows mortality rates for all causes. Nonsmokers reporting moderate exercise are the referent groups. Exercise is inversely related to mortality in males and females in both smokers and nonsmokers. Those who answered "none" had by far the highest rates. Figure 5 shows relative risks for cancer (all sites). Those who answered "none" had highest rate, but in male smokers and female smokers and nonsmokers, the rate was higher in those who reported they exercised heavily than in those who exercised moderately.

Table 4 shows the SMR for selected causes for those reporting heavy exercise. They were below 100 for ischemic heart disease and cerebrovascular disease in both sexes. The SMR also were under 100 for the categories other heart disease (ICD-9 codes 390-405, 415-429), other circulatory diseases (440-459), respiratory dis-
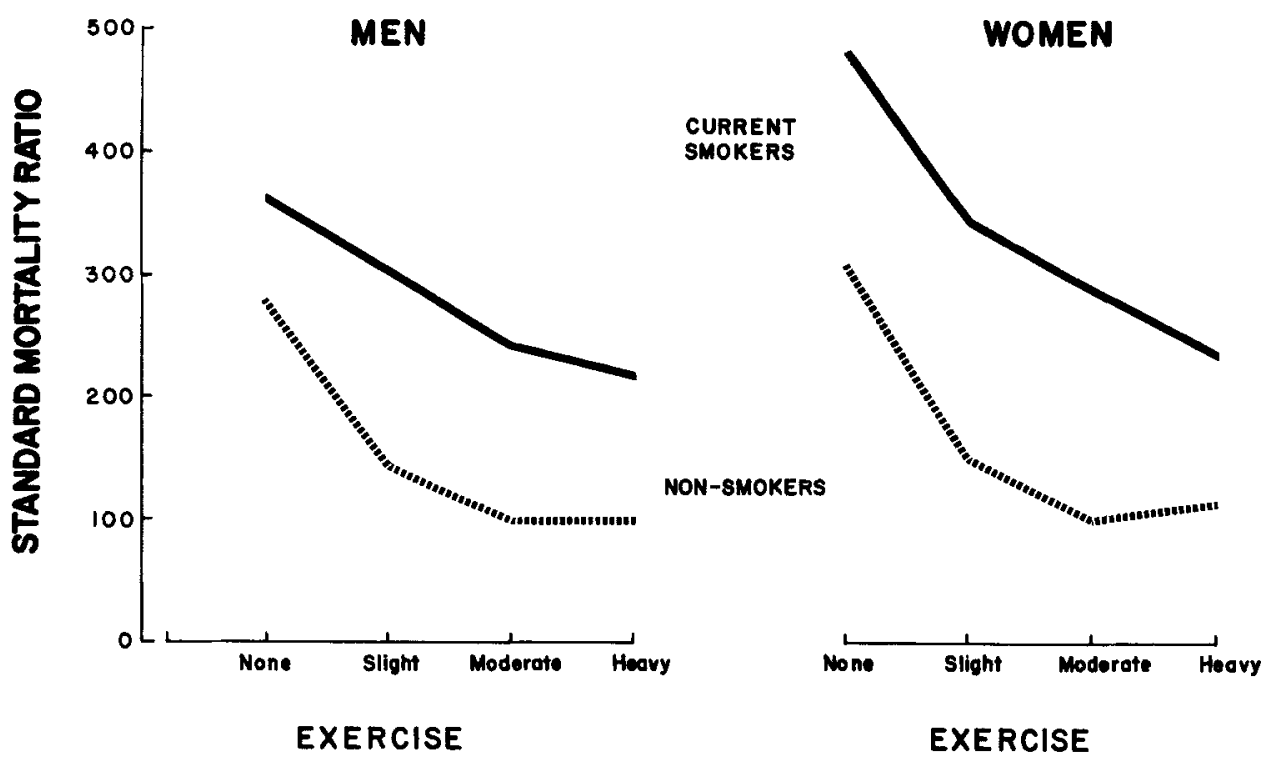

FIG. 4. Standardized mortality ratios by exercise for all causes of death for current smokers and nonsmokers, by sex. 
FIG. 5. Standardized mortality ratios by exercise for cancer (all sites) for current smokers and nonsmokers, by sex.

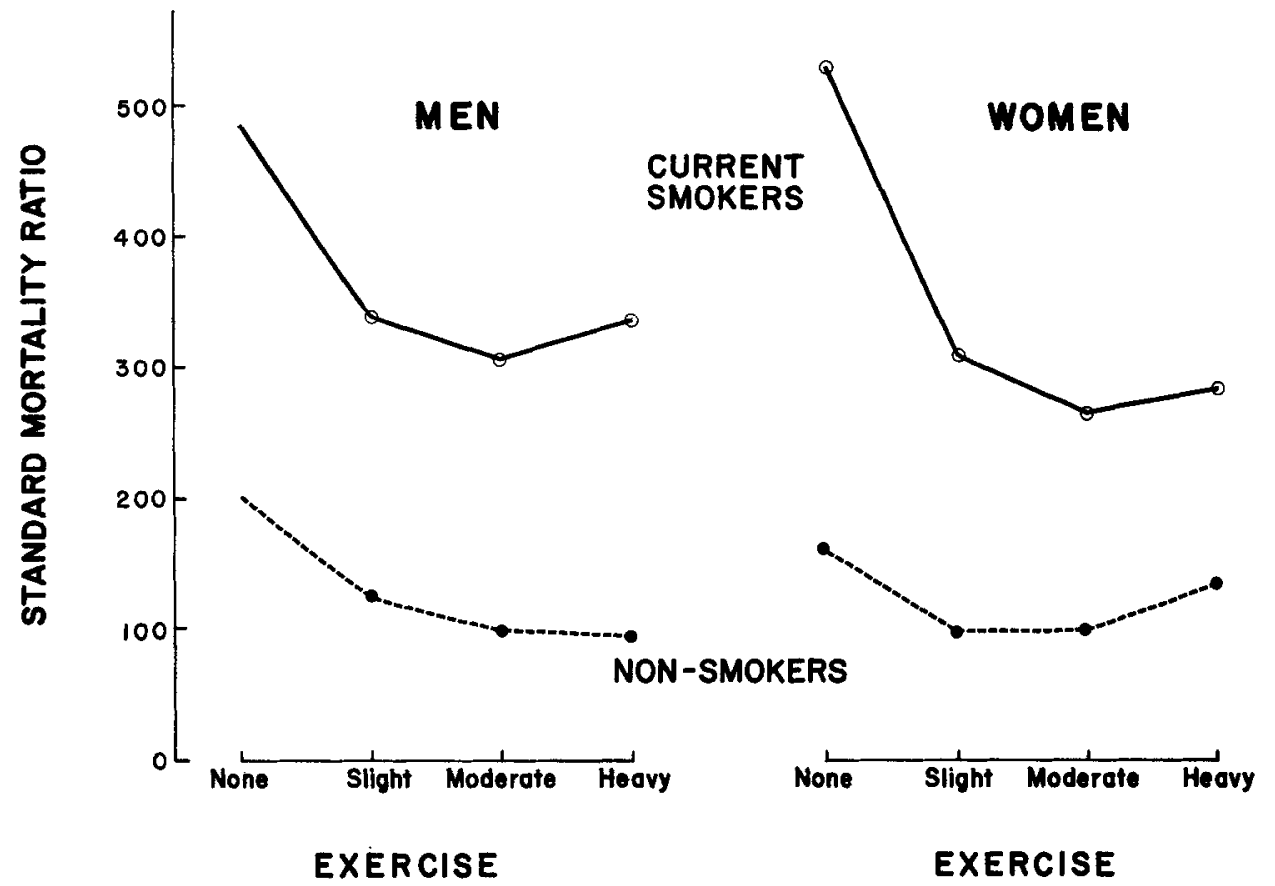

eases (480-519), digestive diseases (531-579), and genitourinary diseases (580-629). Most of these categories (not shown in Table 4) had less than ten observed deaths in subjects who exercised heavily. It was only for the cancer deaths that an increase in SMR for heavy exercisers appeared. There was a $20 \%$ excess in men and $37 \%$ excess in women for lung cancer, colon-rectum cancer, and pancreatic cancer. None of these increases were statistically significant $(P>0.05)$. Other cancer sites in women with five or fewer observed deaths that also showed an increased risk include breast cancer (SMR 123) and uterine cancer (255). In men, digestive system cancers (other than colon-rectum and pancreas) had an SMR of 70 with 11 observed deaths, and prostate cancer had an SMR of 86 with five observed deaths.

\section{Discussion}

In the first 2 years of CPS II more than 19,000 deaths were reported. However, only $38 \%$ of the deaths were in persons who reported on the questionnaire that they were not sick or had a history of heart disease, stroke, or cancer. Sick persons were not analyzed because it was believed their weight was a likely consequence of their disease, rather than a precursor.

The curves for both men and women for all causes of death were $U$ shaped rather than $J$ shaped as in the previous American Cancer Society prospective study and the Society of Actuaries 1979 Build Study. This is almost certainly because of a lingering effect of history of illness in underweight persons who were not excluded from the study with the definitions of illness that were set.

In the 1979 Build Study ${ }^{3}$ it was shown that mortality in the 1st 5 years overstates the long-range mortality data in those $15 \%$ or more underweight by about $10 \%$. For the first 2 years of experience it might be even higher. Women $25 \%$ or more underweight show about a $25 \%$ greater mortality in the first 5 years than for a 20 -year period. Persons $25 \%$ or more overweight have about a $10 \%$ greater mortality in the first 5 years than over a 20 -year period. Therefore the relative mortality figures reported in this study should be considered as preliminary.

TABLE 4. Standardized Mortality Ratios* for Selected Causes for Subjects Reporting Heavy Exercise

\begin{tabular}{lccccc}
\hline & \multicolumn{2}{c}{ Men } & & \multicolumn{2}{c}{ Women } \\
\cline { 2 - 3 } \cline { 5 - 6 } \multicolumn{1}{c}{$\begin{array}{l}\text { No. of } \\
\text { Cause of death }\end{array}$} & deaths & SMR & & $\begin{array}{l}\text { No. of } \\
\text { deaths }\end{array}$ & SMR \\
\hline All causes & 395 & 92 & & 137 & 94 \\
Ischemic heart disease & 132 & 88 & & 22 & 64 \\
Cerebrovascular & 16 & 80 & & 12 & 87 \\
$\quad$ disease & 136 & 99 & & 67 & 120 \\
All cancer & 58 & 119 & & 16 & 136 \\
Lung cancer & 17 & 122 & & 10 & 137 \\
Colorectal cancer & 12 & 122 & & 7 & 137 \\
Pancreatic cancer & & & & & \\
\hline
\end{tabular}

SMR: standardized mortality ratio.

* Referent category is persons exercising moderately. 
The current study showed that persons of average weight (both male and female) had the lowest mortality. This also is in contrast to the Build Study ${ }^{3}$ and first American Cancer Society Study which showed the lowest risk (SMR 96) was in women $10 \%$ to $20 \%$ below average weight. In men in the 80 to 89 pounds relative weight category the SMR was 105 . In this study the SMR for this category were 116 and 126 in women and men, respectively.

A letter to the editor ${ }^{4}$ about the Manson et al. ${ }^{1}$ report on body weight and longevity commented that physical activity is highly correlated with body weight and should be considered in any analysis of body weight and mortality. In this study a separate analysis of mortality by relative weight restricted to subjects who reported they exercised moderately or heavily showed virtually the same pattern as for all subjects, although mortality was at a lower level and SMR for underweights and overweights were lower.

An unexpected finding in this analysis was the elevated risk of cancer of the lung, colon-rectum, and pancreas for subjects who reported heavy exercise. These increases were small and not statistically significant. Whether or not the observed increased SMR are real will have to await future follow-up studies.

\section{REFERENCES}

1. Manson JE, Stampfer MJ, Hennekens $\mathrm{CH}$, Willett WC. Body weight and longevity. JAMA $1987 ; 257: 353-358$.

2. Lew EA, Garfinkel L. Variations in mortality by weight among 750,000 men and women. $J$ Chron Dis 1979; 32:563-576.

3. Build Study 1979. Society of Actuaries and Association of Life Insurance Medical Directors of America, 1980.

4. Beasley JW. Body weight and longevity (Letter). JAMA 1957; $257: 1895$. 